\title{
战略决策专家系统中的“分解一综合” 知识表示法
}

\author{
涂承宇 \\ 喻学恒 \\ (海军装备论证研究中心,北京) (海军工程学院, 武汉)
}

\section{关链词专家系统、决策支持系统、思维科学}

决策专家系统是用来完成决策任务的专家系统. 研究人在决策过程中的行为特性, 对于 建立决策专家系统具有重要的指导意义. 现有的决策科学, 或从心理模型的角度来研究不同 外界条件对人的决策行为的影响, 如文献 $[1-3]$, 或从数学的角度探讨如何进行规范化决策, 避免人在决策过程中的失误，如文献 $[4,5]$. 这些方法都没有从方法论的角度剖析人在决策过 程中的行为特性, 因而对建立决策专家系统缺乏直接的指导意义. 笔者首次从这个角度研究 了人在决策过程中的以下特性,为建讧决策专家系统提供依据:

1. 问题求解的“分解一综合”特性 (简称 DS 特性)；2. 非单调推理特性；3. 基于模糊蔇率 的推理特性； 4. 自学习特性； 5 . 功能分布特性；6. 层次特性； 7. 问题分布式求解特性. 其中 DS 特性是笔者提出的.

决策问题是一个很复杂的问题,需要考虑的问题很多. 人脑的推理能力有限, 若一下子将 所有约束都考虑进去,必然会觉得无从下手. 一个有经验的决策者, 不是一上来就把所有的因 索都考虑进来, 而是把问题分解成若干中介问题, 先解决中介问题, 再考虑源问题。一般地, 源 问题不一定是它的中介问题的简单组合，这或是由于中介问题不是相互独立的，或是由于中介 问题的组合不能覆盖源问题. 因此, 人们解决中介问题以后, 还要对中介问题的解进行调整淙 合,才能得到源问题的满意解. 中介问题的选定满足下述条件:

1）对其中某些中介问题,决策者具有丰富的决策经验,可有效地解决.

2) 中介问题所含的约束条件较少,比源问题容易解决.

定义人们在问题求解过程中, 将源问题分解为若干中介问题分别求解, 再通过综合平 衡中介问题的解来求解源问题的问题求解策略,叫作 DS 特性.

例 1. 电力发展规划的确定是一个很复杂的战略决策问题. 它受国家水力、煤炭、石油等资 源条件,工农业生产水平, 工农业发展规划, 国家财政情况等诸多因素的影响. 如果我们将约 束条件分为两大类, 先从“需要”和“可能”两个方面考虑, 则决策方案的确定相对容易些. 一 般, 常出现需要大于可能的情况. 因此, 还存在一个综合平衡的过程, 以协调两者间的矛盾. 这 里,“需要”和“可能”可作为电力发展规划的两个中介问题, 而后者是前两者的源问题.

笔者定义的 DS 特性,并非只体现在决策过程中. 大系统的分块优化方法, 用变换反变 换的方法简化计算, 甚至目标递归求解策略, 都是 DS 特性的体现. 凡是在难于直接求解的 场合, 人们的思维都表现出 DS 特性. DS 行为特性是人在进行高维思维时的常见特性.

本文 1988 年 7 月 1 日收到. 
专家系统的性能，除了受所采用的专嫁知识的水平的影响以外，还深受知识表示方式的影 响. 专家系统应采用基于知识的表示方法 (Knowledge-Based Approach)。其形式应接近专 家吙识的自然形式. 这样有利于提高专家系统的性能.

战略决策是高维决策. 人们的思维在战略决策中频繁地表现出 DS 特性. 为此, 笔者提 出了“分解一综合”知识表示法,简称 DS 表示法.

DS 表示法可用一种超图表示,其一般形式如图 1 所示. 图中的节点表示待求解的问题; 纵向的弧线表明问题分解的关系(即 DS 表示法的 D 部分), 弧线的下端的问题是弧线上端问 题的中介问题(如: 问题 $B 1 \sim B k$ 是问题 $A$ 的中介问题); 横向的弧线表示对中介问题解的 综合平衡过程(即 DS 表示法的 $S$ 部分. 如 $S-A$ 弧线, 表示对中介问题 $B 1 \sim B k$ 解的综 合平衡过程).一个问题可以有多个中介问题, 也可以只有一个中介问题. 不同的问题可以有 公共的中介问题。无论是哪一种情况，综合乎衡过程都不可缺省。

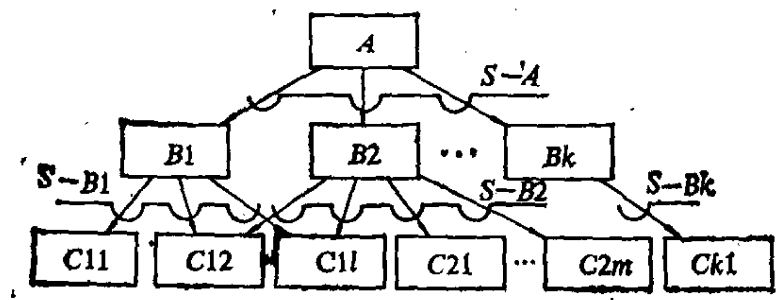

图 1 DS 表示法的一般形式

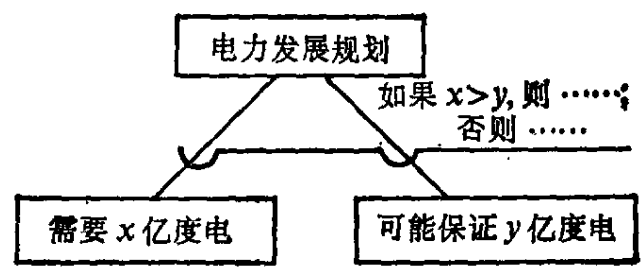

图 2 DS 表示法的一个实例

例 2. 利用 DS 表示法,可将例 1 的知识表示成图 2 的形式.

笔者通过充分的分析论证,得出若干有关 DS 表示法的结论:

1. 与/或图表示法是 DS 表示法的特例, 且存在能够用 DS 表示法表示而不能用与/或图 表示法表示的知识. 因此, DS 表示法比与/或图表示法具有更强的表示能力.

2. DS 表示法便于大幅度提高系统的运行效率.

DS 表示法可以比 MYCIN 系统的 UPDATEDBY 表 ${ }^{[6]}$ 更有效地限制允许访问的规则空 间（即进行规则匹配时的候选规则空间）从而更便于提高系统的运行效率. 由于 DS 特 性是一种常见的行为特性, 因此, 应用 DS 表示法的专家系统, 常常比 MYCIN 那样用 UPDATEDBY 表方法的专家系统运行效率高.

3. DS 表示法支持多种问题求解模型. 包括：1）支持正向和反向推理模型；2）支持目 标递归求解模型；3）支持日程表模型；4）支持黑板㷬型.

4. DS . 表示法支持元级推理.

5. DS 表示法便于构造高能解解释系统.

DS 表示法反映了人的 DS 行为特性,因而便于解释元级知识, 从而便于用户理解系统的 推理过程. 例如, 对于图 1 中问题 $A$ 的求解过程解触倠可以分两步进行: 首先, 系统告诉用 户, 问题 $A$ 的解是综合考虑中介问题 $B 1 \sim B k$ 的解的结果. 即先告诉用户求解 $A$ 的思路, 然. 后再告诉用户由这些中介问题的解推出问题 $A$ 的解的具体过程. 这种解释方法, 使用户对问 题 $A$ 的求解思路先有个概略的了解，再考虑求解的细节. 既便于用户理解一些复杂的推理，又 便于舍弃一些对于用户来说无关紧要的或团户已经熟知的细节, 加快解释的进程, 突出解释的 重点. 使不同水平的用户都可以得到足够详细的解释，又不觉得过分罗嗦.

6. DS 表示法便于获取专家知识. 
MYCIN 及其衍生系统, 都是被动地接受一条条规则, 往往使人们觉得过于零乱, 没有一个 整体概念. DS 表示法反映了人的 DS 行为特性, 这使得基于 DS 表示法的专家系统可以先 获取专家们的解题思路, 然后再获取更详尽的知识, 使知识获取工作显得比较自然, 并带有一 定的启发性.

7. DS 表示法支持非单调推理.

8. DS 表示法模块性好, 便于更新知识.

9. DS 表示法支持问题分布式求解.

笔者已成功地将 DS 表示法用于战略决策专家系统中: 构造了基于 DS 表示法的咨询 程序、知识获取程序、解释程序以及推理机. 也可以说, 笔者构造了具有 DS 风格的元专家系 统. 此外,由于 DS 特性并非只体现在决策过程中, 故 DS 表示法具有较普遍的适用性. 实 践证明,笔者关于人在决策过程中行为特性的分析是正确的,提出的 DS 表示方法是成功的.

\section{参考文献}

[1] Sage, A. P., IEEE Trans. Systcm, Man and Cybernatics, 11(1981), 640-678.

[2] Simon, H. A., Alministrative Bchavior: A Study of Adecision-making Processes in Administrative Organization, 2 ed., MacMilian, New York. 1957.

[3] Janis, I. L. \& Mann, L., Decision Making, Free Press, 1976.

[4] Berger, J. O., Staristical Decision Theory: Foundations, Concepts and Methods, Springer-Verlag, New York, 1980.

[5] Chankong, V. \& Haimes, Y. Y., Multiobjective Decision Making: Theory and Mezhodology, North-Holland. New York, 1983.

[6] Buchanan, B. G. \& Shortliffe, E. H. (ed.). Rule Bascd Expert System: the MYCIN Experiments of the Stanford Heuristic Programming Project, Addison-Wesiey, Reading, Mass., 1984. 